(C) [2008] IEEE. Reprinted, with permission, from Edgar, J.A. ; Zareie, H.M. ; Blaber, M. ; Dowd, A. ; Cortie, M.B. Synthesis of hollow gold nanoparticles and rings using silver templates. International Conference on Nanoscience and Nanotechnology, 2008. ICONN 2008. 25-29 Feb. 2008. This material is posted here with permission of the IEEE. Such permission of the IEEE does not in any way imply IEEE endorsement of any of the University of Technology, Sydney's products or services. Internal or personal use of this material is permitted. However, permission to reprint/republish this material for advertising or promotional purposes or for creating new collective works for resale or redistribution must be obtained from the IEEE by writing to pubs-permissions@ieee.org. By choosing to view this document, you agree to all provisions of the copyright laws protecting it 


\title{
Synthesis of Hollow Gold Nanoparticles and Rings Using Silver Templates
}

\author{
Jonathan A. Edgar, Hadi M. Zareie, Annette Dowd and Michael B. Cortie \\ Institute for Nanoscale Technology, University of Technology Sydney, Australia \\ Email: jonathan.edgar@student.uts.edu.au
}

\begin{abstract}
Gold nanoshells have gained attention recently due to their interesting optical properties. In particular, their spectrally selective extinction has been exploited for experimental therapeutic medical applications, spectral applications and contrast enhancement for analytical techniques (e.g. Surface Enhanced Raman Scattering Spectroscopy, SERSS). Synthesis of hollow Au/Ag alloy particles utilising galvanic replacement are presented. Ag template particles stabilised by the surfactant CTAB (hexadecyltrimethyl ammonium bromide) produce a distinct population of nanorings upon addition of excess $\mathrm{HAuCl}_{4}$. Nanorings present an interesting particle geometry, with optical properties exhibiting characteristics of nanorods and nanoshells.
\end{abstract}

\section{INTRODUCTION}

Two main classes of nanoshells dominate current literature: core-shell and hollow nanoparticles. Nanoshells optical response may be tuned by adjusting the ratio of the inner diameter to the outer diameter. This can be achieved by varying the template particle radius or increasing the thickness of the metal shell.

Core-shell particles consist of a dielectric core (e.g. inorganic or polymer) sheathed by a thin metallic layer. Core-shell particles were introduced by Zhou et. al. in 1994 where $\mathrm{Au}_{2} \mathrm{~S}-\mathrm{Au}$ (core-shell) nanoparticles were shown to have red-shifted optical response initially attributed to quantum confinement due to the dimensions of the thin shell [1]. However, Averitt et. al. provided evidence that for the case of metal shells quantum confinement effects would broaden the extinction spectrum but not shift the maxima [2]. Additionally, Averitt et. al. also suggested an alternative formation mechanism where gold nucleates on core particles and in solution, which is supported by kinetics data measured by spectrometry. This is opposed to the original diffusive reduction of $\mathrm{Au}_{2} \mathrm{~S}$ core particles, presented by Zhou et. al. which ultimately resulted in solid gold particles.

Silica core nanoshells represent another architecture and growth process for core shell nanoparticles, popularized by Halas et. al. [3-5]. Whereby, silica nanoparticles are functionalized with an aminosilane molecule to facilitate attachment of small metal seed particles (typically silver or gold). Additional metal is then electrolessly deposited to form a continuous shell. Polymeric core particles (e.g.) have also been utilized as templates in this procedure [6].

In contrast, hollow nanoparticles are made by galvanic replacement of a metallic template nanoparticle of lesser standard redox potential. For gold nanoshells template particles of silver [7-9] and cobalt [10] have been demonstrated.

Structure of the hollow particle is dependent on the template particle, i.e. for any shape of template particle a hollow analogue is possible [9]. The resulting nanoshell is an alloy of gold and silver, where excess addition of tetrachloroauric acid results in the replacement of remaining silver resulting in reconstruction and formation of 'pinholes', ultimately leading to fragmentation (figure 1).

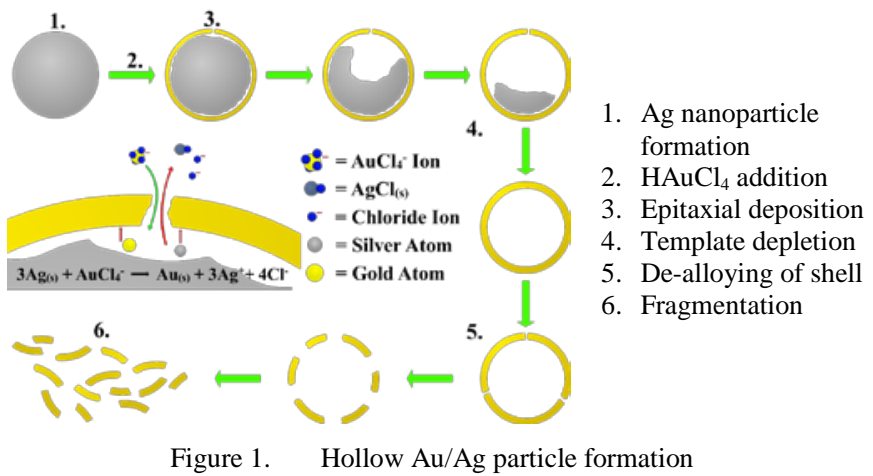

Triangular nanorings have been synthesized by the galvanic replacement reaction using silver triangles as a template [11]. Colloidal lithography has also been used to synthesise nanorings [12]. Briefly, polystyrene spheres are dispersed on a substrate by electrostatic self-assembly. A 20 $-40 \mathrm{~nm}$ gold film is then deposited at normal incidence with respect to the substrate. Argon beam ion etching is then used to remove the gold film. During the etch process secondary sputtering creates the ring structure around the base of the polystyrene spheres. Finally, the spheres are removed by UV-ozone treatment leaving free-standing ring structures.

Due to the nature of the optical properties and structure of nanoshells and nanorings a broad range of applications may benefit from the use or inclusion of these particles. Nanoshells, in particular, have already demonstrated versatility with experimental validation for diagnostic and therapeutic medical applications [13-15], surface enhanced Raman spectroscopy [16, 17] and chemical [4, 18, 19] and biosensors [20, 21]. Nanorings can also provide significant enhancements in these applications with proof-of-concept for sensing applications [22]. In addition to being interchangeable with nanoshells, nanorings also exhibit polarization dependent optical properties. 


\section{EXPERIMENTAL PROCEDURE}

Silver nanoparticles ( $\sim \mathrm{nm}$ in diameter) have been used as templates for the galvanic replacement reaction. Template particles are synthesised by borohydride reduction of $\mathrm{AgNO}_{3}$ in the presence of CTAB. Gold metal is epitaxially deposited on the surface of the template by heating the silver particles for 15 minutes at $75^{\circ} \mathrm{C}$ then dropwise addition of $1 \mathrm{mM}$ $\mathrm{HAuCl}_{4}(5-10 \% \mathrm{v} / \mathrm{v})$

Calculations were performed using an implementation of the discrete dipole approximation, DDSCAT. The discrete dipole approximation is used to calculate absorption and scattering properties of a target using an array of polarisable point dipoles. An inter-dipole spacing (IDS) $<1 \mathrm{~nm}$ is satisfactory for most cases, confirmed in this case by the convergence of the absorption efficiency. A torus was used for the calculation of the optical properties of the nanorings. A comparison of DDA with Mie theory using a $49 \mathrm{~nm}$ radius gold sphere in vacuum with an IDS of $2.5 \mathrm{~nm}$ and 24464 occupied lattice sites gives $\mathrm{Q}_{\mathrm{abs}}$ values of 3.3 and 3.2 at a peak position of $520 \mathrm{~nm}$ (DDA and Mie respectively)

\section{RESULTS AND DISCUSSION}

Formation of the Au/Ag alloy nanoshell occurs at the expense of silver metal, as favoured by the standard reduction potentials $(0.99 \mathrm{~V}$ and $0.80 \mathrm{~V}$ vs standard hydrogen electrode, Au and Ag respectively). Using template particles of such small diameter presents difficulties for achieving high yields, where larger diameter particles are less troublesome [8]. Upon addition of excess $\mathrm{HAuCl}_{4}$ (step 5 , figure 1 ), pin-hole formation is induced. This is due to dealloying of the nanoshell which results in cage-like structures and ultimately fragmentation. Figure 2 shows a mix of cagelike and nanoring structures.

We have found that a distinct population ( $10 \%$ ) of gold nanorings results when we use CTAB-stabilised template particles in conjunction with excess tetrachloroauric acid (figure 2). It is uncertain as to the formation mechanism of these nanorings. However, it is known that CTAB binds preferentially to (010) and (001) gold crystallographic facets [23]. This gives the possibility of preferential binding at the 'equator' of the forming hollow nanoparticle. Shielding of the 'equator' of the shell could expose the 'poles' resulting in localised reconstruction and "pin-hole” formation.

DDSCAT was used to approximate the extinction properties of nanorings of varied aspect ratio (figure 3 ). Aspect ratio is defined as the ratio of the outer diameter (OD) to the ring thickness. From figure 3 it can be seen that nanorings exhibit two plasmon modes. A fixed low intensity peak at $510 \mathrm{~nm}$ corresponds to oscillations across the plane of the nanoring. The dominant peak of the extinction spectrum is attributed to oscillations within the plane and is proportional to the aspect ratio.

Intensities in figure 3 may be inaccurate due to the size of the nanorings and exclusion of surface scattering effects in DDSCAT. The mean free path of electrons in gold and silver is of the order of $40-50 \mathrm{~nm}$ [24]. Oscillating electrons in nanoparticles smaller than this distance will suffer plasmon
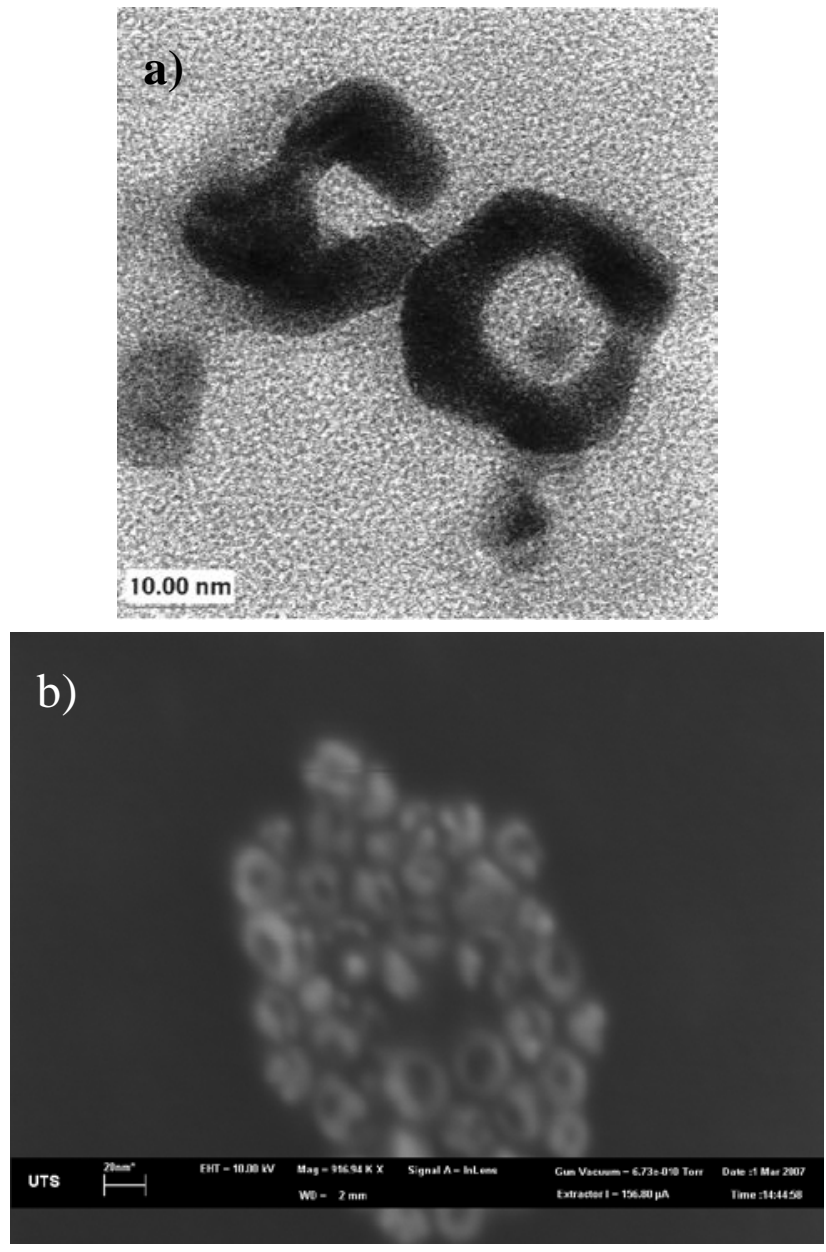

Figure 2. a) TEM of gold nanorings

b) SEM of gold nanorings (scale bar $20 \mathrm{~nm}$ )

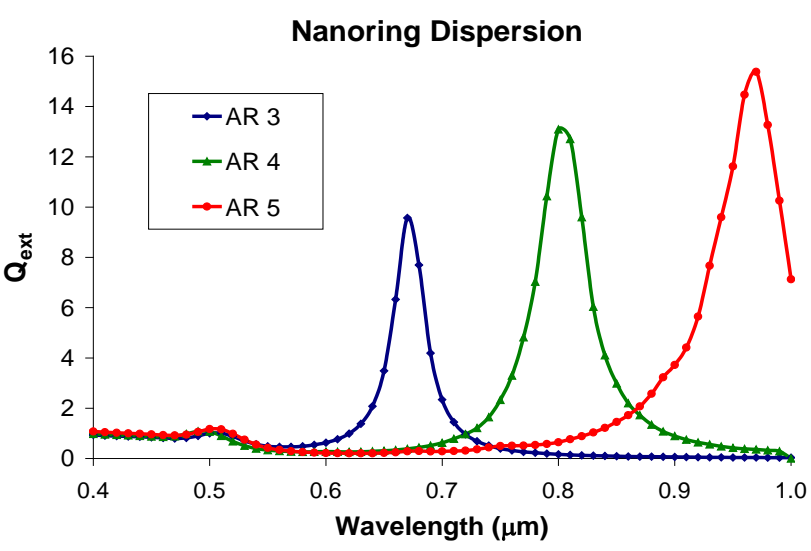

Figure 3. Calculated (DDSCAT) extinction spectra for gold nanorings

damping due to surface scattering events which reduces the intensity of the absorption and increases the peak width. Despite the thickness of each ring being $10 \mathrm{~nm}$ the effective mean free path is slightly greater and can be approximated using an expression determined for nanoshells by Granqvist et. al. [25] (as cited by Mulvaney [26]). The effective mean 
free path for each nanoring is 12.6, 14.4 and $15.9 \mathrm{~nm}$ for aspect ratio 3, 4 and 5 nanorings respectively.

Nanorings can be either dependent or independent of polarization depending on their orientation with respect to incident light. Nanorings dispersed on a two dimensional substrate oriented with their plane parallel to the substrate (i.e. flat) will be independent of the polarization of incident light (for an angle of incidence of $90^{\circ}$ ). However, for rings oriented perpendicular to the substrate the transverse and planar plasmon modes may be excited selectively by changing the polarization.

\section{CONCLUSION}

Formation of hollow nanoparticles from $5 \mathrm{~nm} \mathrm{Ag}$ template particles is possible but will not yield as high tuneability as that shown for template particles of greater diameter. However, subsequent formation of nanorings presents an intriguing development. Gold nanorings are shown to have an optical response with characteristics of both nanorods and nanoshells. The unique property of selective polarization dependence presents an additional permutation for the application of plasmonically active anisotropic nanoparticles.

\section{ACKNOWLEDGMENTS}

The authors wish to acknowledge the support of the ARC under linkage project LP0669111 in collaboration with AGR Matthey and assistance from the Electron Microscopy Unit of Sydney University.

\section{REFERENCES}

[1] H. S. Zhou, I. Honma, H. Komiyama, and J. W. Haus, "Controlled synthesis and quantum-size effect in gold-coated nanoparticles," Phys. Rev. B, vol. 50, pp. 12052 - 12057, 1994.

[2] R. D. Averitt, D. Sarkar, and N. J. Halas, "Plasmon resonance shifts of Au-coated $\mathrm{Au}_{2} \mathrm{~S}$ nanoshells: insight into multicomponent nanoparticle growth," Phys. Rev. Lett., vol. 78, pp. 4217 - 4220, 1997.

[3] S. W. Bishnoi, C. J. Rozell, C. S. Levin, M. K. Gheith, B. R. Johnson, D. H. Johnson, and N. J. Halas, "All-optical nanoscale pH meter," Nano Letters, vol. 6, pp. 1687 - 1692, 2006.

[4] N. J. Halas, S. Lal, P. Nordlander, J. B. Jackson, and C. E. Moran, "Nanoparticle-based all-optical sensors," vol. 6778316. United States: William Marsh Rice University, 2004.

[5] S. J. Oldenburg, R. D. Averitt, S. L. Westcott, and N. J. Halas, "Nanoengineering of optical resonances," Chem. Phys. Lett., vol. 288, pp. 243 - 247, 1998.

[6] W. Shi, Y. Sahoo, M. T. Swihart, and P. N. Prasad, "Gold nanoshells on polystyrene cores for controls of surface plasmon resonance," Langmuir, vol. 21, pp. 1610 - 1617, 2005.

[7] Y. Sun, B. T. Mayers, and Y. Xia, "Template-engaged replacement reaction: a one-step approach to the large-scale synthesis of metal nanostructures with hollow interiors," Nano Letters, vol. 2, pp. 481 485, 2002.
[8] Y. Sun and Y. Xia, "Alloying and dealloying processes involved in the preparation of metal nanoshells through galvanic replacement reaction," Nano Letters, vol. 3, pp. 1569 - 1572, 2003.

[9] Y. Sun and Y. Xia, "Mechanistic study on the replacement reaction between silver nanostructures and chloroauric acid in aqueous medium," J. Am. Chem. Soc., vol. 126, pp. 3892 - 3901, 2004.

[10] A. M. Schwartzberg, T. Y. Olson, C. E. Talley, and J. Z. Zhang, "Synthesis, characterisation, and tuneable optical properties of hollow gold nanospheres," J. Phys. Chem. B., vol. 110, pp. 19935 - 19944, 2006.

[11] L.-P. Jiang, S. Xu, J.-M. Zhu, J.-R. Zhang, J.-J. Zhu, and H.-Y. Chen, "Ultrasonic-assisted synthesis of monodisperse single-crystalline silver nanoplates and gold nanorings," Inorg. Chem., vol. 43, pp. 5877 - 5883, 2004.

[12] J. Aizpurua, P. Hanarp, D. S. Sutherland, M. Käll, G. W. Bryant, and F. J. G. d. Abajo, "Optical properties of gold nanorings," Phys. Rev. Lett., vol. 90, pp. 057401 -1, 057401 - 4, 2003.

[13] A. Gobin, M.-H. Lee, N. J. Halas, W. D. James, R. A. Drezek, and J. L. West, "Near-infrared resonant nanoshells for combined optical imaging and photothermal cancer therapy," Nano Letters, vol. 7, pp. 1929 - 1934, 2007.

[14] C. Loo, A. Lin, L. Hirsch, M.-H. Lee, J. Barton, N. Halas, J. West, and R. Drezek, "Diagnostic and therapeutic applications of metal nanoshells," Nanofabrication towards Biomedical Applications, pp. 327-342, 2005.

[15] C. Wu, X. Liang, and H. Jiang, "Metal nanoshells as a contrast agent in near-infrared diffuse optical tomography," Optics Communications, vol. 253, pp. 214-221, 2005.

[16] R. A. Alvarez-Puebla, D. J. Ross, G. A. Nazri, and R. F. Aroca, "Surface-Enhanced Raman Scattering on Nanoshells with Tunable Surface Plasmon Resonance," Langmuir, vol. 21, pp. 10504-10508, 2005.

[17] S. J. Oldenburg, S. L. Westcott, R. D. Averitt, and N. J. Halas, "Surface enhanced Raman scattering in the near infrared using metal nanoshell substrates," J. Chem. Phys, vol. 111, pp. 4729 - 4735, 1999.

[18] G. Raschke, S. Brogl, A. S. Susha, A. L. Rogach, T. A. Klar, J. Feldmann, B. Fieres, N. Petkov, T. Bein, A. Nichtl, and K. Kürzinger, "Gold nanoshells improve single nanoparticle molecular sensors," Nano Lett., vol. 4, pp. 1853 - 1857, 2004.

[19] Y. Sun and Y. Xia, "Synthesis of gold nanoshells and their use in sensing applications," Materials Research Society Symposium Proceedings, vol. 776, pp. 31-36, 2003.

[20] H. T. Beier, C. B. Cowan, I. H. Chou, J. Pallikal, J. E. Henry, M. E. Benford, J. B. Jackson, T. A. Good, and G. L. Cote, "Application of surface-enhanced Raman spectroscopy for detection of beta amyloid using nanoshells," Plasmonics, vol. 2, pp. 55-64, 2007.

[21] J. L. West, N. J. Halas, S. J. Oldenburg, and R. D. Averitt, "Metal nanoshells for biosensing applications," vol. 6699724. United States: William Marsh Rice University, 2004.

[22] E. M. Larsson, J. Alegret, M. Käll, and D. S. Sutherland, "Sensing characteristics of NIR localized surface plasmon resonances in gold nanorings for applications as ultrasensitive biosensors," Nano Lett., vol. 7, pp. 1256 - 1263, 2007. 
[23] J. Pérez-Juste, I. Pastoriza-Santos, L. M. Liz-Marzán, and P. Mulvaney, "Gold Nanorods: Synthesis, Characterisation and Applications," Coord. Chem. Rev., vol. 249, pp. 1870-1901, 2005.

[24] S. Link and M. A. El-Sayed, "Shape and size dependence of radiative, non-radiative and photothermal properties of gold nanocrystals," Int. Rev. Phys. Chem., vol. 19, pp. 409 - 453, 2000.
[25] C. G. Granqvist and O. Hunderi, "Optical absorption of ultrafine metal spheres with dielectric cores," Zeitschrift fuer Physik, vol. 30, pp. 47 - 51, 1978.

[26] P. Mulvaney, "Surface plasmon spectroscopy of nanosized metal particles," Langmuir, vol. 12, pp. 788-800,1996. 